\title{
Commuters en la frontera México-Estados Unidos
}

\author{
Guillermo Arámburo Vizcarra \\ Instituto de Investigaciones Sociales \\ Universidad Autónoma de Baja California
}

\begin{abstract}
Resumen
Este estudio está dirigido al análisis de un sector de la población típicamente fronterizo, comúnmente conocido como "emigrados", "green cards" o "tarjetas verdes". Para efectos de este trabajo se les considera con el nombre con el que son clasificados por el Servicio de Inmigración y Naturalización de los Estados Unidos (SIN), esto es como commuters, que son las personas que tienen autorización legal para residir y trabajar en la Unión Americana; pero que han optado por sólo trabajar en ese país y mantener su lugar de residencia en México. Los trabajadores commuters han actuado en la frontera México-Estados Unidos desde que aparece el control de la línea internacional. Su integración al mercado laboral norteamericano históricamente ha respondido a las necesidades de fuerza de trabajo ahí generadas, así como a la falta de opciones de trabajo en México que sean competitivas con las que se presentan del otro lado de la frontera.
\end{abstract}

Palabras clave: commuters, "green cards", frontera, mercado laboral norteamericano, migración.

\begin{abstract}
This research addresses the analysis of one sector of the border population commonly known as "immigrated" or "green cards"; however, they will be named "commuters", in this research, according to the classification of the Immigration and Naturalization Service of the United States (SIN). It means that those people who have legal authorization to live and work in the United States; but have decided to work in that country, while keeping their residence in Mexico. The commuter workers have been present along the Mexican-American border since the control of the international line was established. Its integration to the American labor market has historically met the needs of labor force of this zone, and also has solved the lack of job options in Mexico, which could be competitive to the job offers at the other side of the border.
\end{abstract}

Keywords: commuters, "green cards", border, American labor market, migration. 


\title{
COMMUTERS EN LA FRONTERA MEXICO-ESTADOS UNIDOS
}

\author{
Por \\ Guillermo Arámburo Vizcarra*
}

Este estudio está dirigido al análisis de un sector de la población típicamente fronterizo, comúnmente conocido como "emigrados", "green cards" o "tarjetas verdes"; otros estudios los conceptualizan como transmigrantes; sin embargo, para efectos de este trabajo se les considera con el nombre con el que son clasificados por el Servicio de Inmigración y Naturalización de los Estados Unidos (SIN), esto es como commuters, que son aquellas personas que tienen autorización legal para residir y trabajar en la Unión Americana, pero que han optado por sólo trabajar en ese país y mantener su lugar de residencia en México. La mayoría de los commuters cruzan a Estados Unidos para trabajar allá y retornan diariamente; algunos una mínima parte, lo hacen sema. nalmente o por temporadas.

El presente trabajo describe resumidamente algunos resultados de la investigación que actualmente se efectúa en el Instituto de Investigaciones Sociales de la Universidad Autónoma de Baja California, denominada "Encuesta sobre transmigración en la Frontera Norte", que expresan una serie de características de los commuters, las cuales permiten aproximarnos, de manera un poco más profunda, al conocimiento de un sector de la población que interactúa intensamente en la frontera México-Estados Unidos.

La principal fuente de información, además de la bibliográfica y documental, fue obtenida a través de una encuesta en la que se aplicaron 666 cuestionarios en nueve municipios fronterizos regionalizados en tres zonas: Zona Oeste, que considera los municipios de Tijuana, Mexicali, San Luis Río Colorado y Nogales; la Zona Centro a Ciudad Juárez; y Zona Este, a Piedras Negras, Nuevo Laredo, Reynosa y Matamoros. En cada zona se distribuyó una cantidad análoga de cuestionarios, cuyo monto está en proporción al total de la población en cada uno de los municipios. La muestra fue aleatoria simple, y las entrevistas se efectuaron en los sitios de cruce fronterizo, entrevistándose en proporciones similares tanto a commuters peatonales como a aquéllos que se transportaban en automóvil.

\footnotetext{
- Investigador de tiempo completo del Instituto de Investigaciones Sociales de la Universidad Autónoma de Baja California.
} 
Otro aspecto que es necesario aclarar es el relativo al volumen de commuters, ya que actualmente es difícil conocer exactamente el número de commuters legales* que existen en la frontera México-Estados Unidos. Los datos más confiables no son recientes, debido a que son producto de un muestreo que realizó el SIN en diciembre de 1969. En dicho estudio se detectó que en el conjunto de la frontera México-Estados Unidos había aproximadamente 49,770 commuters ${ }^{1}{ }^{\text {distribuidos fundamentalmente }}$ en los estados de Texas $(23,339)$ y California $(20,753)$. En el trabajo de Davis S. North y Marion F. Houston se estima que en 1975 existían 51,922 commuters. ${ }^{2}$ Por otra parte, el trabajo de Margullis y Tuiran que examinó el caso del municipio de Reynosa, estimó conservadoramente, que en 1980 el $4.6 \%$ de la población económicamente activa de esa localidad eran commuters. ${ }^{3}$ Al tomar como base los datos recopilados en los estudios antes mencionados, y considerar el crecimiento de la población de los municipios fronterizos de ambos lados de la frontera, y al derivar un porcentaje aproximado de commuters en la composición municipal de la población económicamente activa - precisamente considerando la composición de commuters en la PEA de 1970 y $1980-$, se estima que para 1980 el número de commuters en el total de municipios fronterizos era aproximadamente de $159,083 .^{4}$

\section{A) ¿QUIENES SON LOS COMMUTERS?}

Dentro del marco estrictamente legal es necesario aclarar que el "programa commuters" no está explícito en ninguno de los estatutos de inmigración del gobierno de los Estados Unidos, sino que este programa ha sido sostenido y avalado por una práctica de carácter administrativo, producto de la interpretación que el Servicio de Inmigración y Naturalización de los Estados Unidos ha dado a la ley de 1927, en un contexto de "vecindad amistosa con México y Canadá". De esta forma, lo que se conoce

\footnotetext{
- Se especifica commuters legales porque en la frontera existe también un número indeterminado de commuters ilegales - debido a la crisis económica actual, todo parece indicar que es ereciente-, los cuales son aquéllos que cuentan con pasaporte local ("border crossing card") que no les permite laborar, o que sin disponer de este documento, cruzan la frontera diariamente para trabajar en Estados Unidos. Un caso de commuters "ilegales" se examina en: Arámburo V., Guillermo. Transmigración en la frontera Tijuana-San Diego. El caso de los trabajadores agricolas indocumentados. Tesis, El Colegio de la Frontera Norte, Tijuana, B.C. 1986.

' Inmigration and Naturalization Service, USA Department of Justice and Manpower Administration, U.S. Department of Labor, eitado en: Ericson, Anna-Stina, "The Impact of Commuters on the Mexican-American Border Area". Monthly Review. (August, 1970). p. 18.

"North, S. David and Marion F. Houston. The Characteristics and Role of Illegal Aliens in the U.S. Labor Market: An Exploratory Study. Washington, Linton \& Company, 1976, p. A.9.

${ }^{3}$ Margulis, M. y Rodolfo Tuiran. Desarrollo y Población en la Frontera Norte: el caso de Reynosa. México, El Colegio de México. 1986.

- Una explicación más amplia de la metodología utilizada para obtener esta cifra se encuentra en el reporte de la investigación Encuesta sobre transmigración en la frontera norte, que publicará el Instituto de Investigaciones Sociales de la Universidad Autónoma de Baja California.
} 
como programa commuters, es sólo la aceptación administrativa de una actividad que ha evolucionado en forma natural y que sólo posee un disfraz legislativo; además, es una práctica que el SIN ha permitido que funcione por más de sesenta años. La definición aceptada por el SIN sobre este programa, describe y califica su verdadero carácter: "La situación del commuter no entra en ninguna categoría precisa que se pueda encontrar en los estatutos migratorios. Su estatus es artificial, sostenido con base en las relaciones compartidas entre vecinos amistosos". ${ }^{5}$

El mismo SIN nos proporciona una definición muy clara de lo que es un commuter: "Aquel extranjero a quien se ha otorgado legalmente el privilegio de residir permanentemente en los Estados Unidos pero escogió residir en territorio extranjero contiguo y conmutar a su lugar de empleo en los Estados Unidos".6

En 1969, un reporte de la Comisión de Derechos Civiles del Congreso intitulado "The Commuter on the United States-Mexico Border", ${ }^{7}$ iniciaba sus notas de pie de página marcando las diferencias entre los commuters de Canadá y los de México, manifestando que: "Los commuters canadienses no deprimen las condiciones económicas de las localidades fronterizas - como las deprimen los commuters mexicanos-, porque ellos viven (los canadienses) con costos económicos de vida sustancialmente idénticos; trabajan en lugares donde hay alto índice de sindicalización, y ellos mismos están también altamente sindicalizados. $\mathrm{O}$ sea que, son bien asimilados en la fuerza de trabajo y no representan una competencia desleal a los trabajadores de la Unión Americana". ${ }^{8}$

En esos años la situación de los commuters mexicanos fue muy cuestionada, puesto que efectivamente fueron utilizados como rompehuelgas en los campos agrícolas donde la "Unión Farm Workers" tenía sindicalizada a la fuerza de trabajo. La posición de Dolores Huerta -precisamente de esta organización-, durante los debates del Congreso dejó un precedente al respecto, oponiéndose reiteradamente a esta práctica migratoria.

La oposición al programa de commuters también presentó diferencias regionales, observándose el más alto grado de oposición en el estado de Texas y mínimamente en Arizona y Nuevo México; en California hubo cierta oposición en el Valle Imperial, en donde la mayoría de los trabajadores laboraban en la agricultura; y en San Diego se presentó muy poca resistencia debido a que la mayoría de los commuters estaban sindicalizados. ${ }^{9}$ La mayor aversión al programa se registró por parte de los líderes de

${ }^{5}$ Report of the Select Commisaion on Western Hemisphere Inmigration. Washington. 1968. p. 102. 6 Ibid. p. 101.

7 "The Commuter on the United States-Mexico Border" United States Commission on Civil Rights. Staff Report. Hearings before the special subcommitte on Labor House Representative: Ninety First Congress; Girst Session on H.R. Washington, D.C., July, 1969.

Ibid. p. 156.

9 Report of the Seleet Commission. . . Op. cit. p. 106. 
organizaciones de méxico-americanos, principalmente los de San Antonio, Texas; estos líderes se reunieron con representantes del Departamento del Trabajo en 1966, y para 1967 todo indicaba que el problema sería incluido en la agenda del Congreso para ese año. Esto dio como resultado que el Departamento del Trabajo de los Estados Unidos dictara algunas restricciones dirigidas especialmente a los commuters; éstas consistian básicamente en la prohibición para que se les contratara en aquellos lugares donde existiera un conflicto laboral (huelgas), y en zonas donde afectaran los niveles salariales y de empleo (condiciones de trabajo). ${ }^{10}$ Es necesario agregar que la instrumentación de est as restricciones no sellevó a cabo porque el Departamento del Trabajo no disponia de los elementos legales para restringir el empleo a los residentes permanentes que ya habían sido admitidos en Estados Unidos, sino sólo a los de nuevo ingreso. 11

A finales de los sesentas el gobierno norteamericano, por medio del Departamento de Estado, resolvió a favor del programa, y sostuvo que la interrupción del mismo afectaría notablemente, por una parte, la relación de vecinos amistosos que ha mantenido con México y Canadá; y por otra, perjudicaría a las localidades fronterizas de ambos lados de la frontera. Por ello, el gobierno de W ashington se manifestó respetuoso de esta "amable ficción" - dentro de los estatutos legales-, como la definió Charles Gordon del Departamento de Justicia de Estados Unidos. ${ }^{12}$

\section{B) EL COMMUTER: UN ACTOR SOCIAL DE FRONTERA}

En una región de colindancia de fronteras internacionales de dos paises, con grados de extrema desigualdad - profundizada por la crisis económica actual-, resulta importante y por demás interesante, examinar el conjunto de situaciones de índole diverso que conforman la cotidianidad fronteriza. Es conveniente precisar en forma breve, que el área fronteriza no es homogénea - aunque la vecindad con los Estados Unidos le imprime un sello a toda la zona-, sus regiones son diferentes: el noroeste, tanto del lado norteamericano como mexicano, está más desarrollado que el noreste, y la interacción fronteriza es más dinámica en el primero que en este último. ${ }^{13}$ Las diferencias de tipo cultural también modifican el panorama, los municipios del extremo este tienen una

\footnotetext{
${ }^{10}$ Ibid. p. 107.

"Ibid. p. 108.

${ }_{12}$ Gordon, Charles, "The Amiable Fietion-Alien Commuters Under Our Inmigration Laws". Hearings before the special. . Op. cit. p. 106.

${ }^{13}$ Diversos estudios han examinado estas diferencias; uno de ellos es el de Clement, Norris. "Perspec. tiva sobre el desarrollo económico de la región fronteriza del suroeste de Estados Unidos". En: Administración del desarrollo de la frontera norte. Ed. Mario Ojeda, México. El Colegio de México. 1982. pp. 114-181. Otroes "The erest of the wave: California Economy". The Economist. May 19.1984. pp. 3-22.
} 
historia más vinculada al conjunto nacional que a los Estados Unidos, en cambio los municipios del extremo oeste son más jóvenes, su historia es más reciente y su desarrollo ha sido de franca dependencia con el vecino del norte; esto es, que hasta hace relativamente poco tiempo el mercado regional del noroeste presentaba una mayor articulación con el mercado de California que con el nacional; sin embargo, con la abrupta devaluación del peso, la vinculación con este último se ha aumentado y con el primero se ha reducido. Este proceso es característico de toda la región fronteriza pero ha impactado en grados distintos al este $y$ al oeste.

Estas diferencias permiten matizar, de forma muy general, el caso de los commuters según la región de la frontera en la que se desenvuelven; asimismo, permiten afirmar, a manera de hipótesis, que los trabajadores de la región este de la frontera tienen condiciones de vida y de trabajo más desfavorables que los del centro y del oeste. Por otra parte, no resulta exagerado sostener que este actor social tiene un estatus privilegiado entre la población fronteriza de México - considerando como indicador principal el ingreso-, ya que por la disparidad peso-dólar sus ingresos se han multiplicado al compás de la devaluación de nuestra moneda.

El ingreso es un indicador adecuado para contrastar en parte las aseveraciones expuestas. En torno a las diferencias regionales, encontramos en el grupo de más bajos ingresos (comprende a las personas que cuentan con una percepción mensual hasta de 300 dólares) que la región oeste sólo concentró al $1 \%$ del total, el centro al $3.6 \%$, y el este al $4.2 \%$. El grupo de los que reciben de 351 a 550 dólares mensuales presenta un comportamiento similar, puesto que el oeste concentra al $2.7 \%$, el centro al $7.2 \%$, y el este al $9.6 \%$ del total de commuters, esto es, 3.5 veces más en la zona este que en la oeste. En cambio, en los grupos de altos ingresos el comportamiento es inverso; la región oeste registra el mayor número de commuters de este grupo en relación a las regiones centro y este; por ejemplo, considerando todos los grupos con ingresos de 951 hasta 2550 dólares mensuales, tenemos que en el oeste se registra el $14.3 \%$ del total, en el centro el $6.3 \%$, y en el este al $4.8 \%$.

Las diferencias son evidentes, los commuters de California tienen mayores ingresos que los del estado de Texas (ver cuadro 1).

La importancia de los trabajadores agrícolas es aún vigente y sigue confirmando que los trabajadores migrantes commuters o transmigrantes son, y han sido, un sector particular de la fuerza de trabajo mexicana que respoude a las necesidades del capital norteamericano. ${ }^{14}$ No en vano los sectores que apoyaron el programa de commuters durante la parte final del decenio de los sesenta, fueron fundamentalmente los hombres

\footnotetext{
14 Bustamante, Jorge A. Espaldas mojadas: materia prima para la expansión del capital norteamericano. México. El Colegio de México, 1976 (Cuadernos del CES. 9).
} 
CUADRO 1. Número y porcentaje de commuters según región (1, 2 y 3 ) y frontera norte total por grupo de ingreso mensual.

\begin{tabular}{|c|c|c|c|c|c|c|c|c|c|c|c|}
\hline $\begin{array}{l}\text { INGRESO REGION } \\
\text { MENSUAL }\end{array}$ & $\begin{array}{l}\text { NUMERO } \\
\text { CASOS }\end{array}$ & $\begin{array}{c}1 \\
\% \\
\text { REGION }\end{array}$ & $\stackrel{\%}{\%}$ & $\begin{array}{l}\text { NUMERO } \\
\text { CASOS }\end{array}$ & $\begin{array}{c}2 \\
\% \\
\text { REGION }\end{array}$ & $\stackrel{\%}{\text { TOTAL }}$ & $\begin{array}{l}\text { NUMERO } \\
\text { CASOS }\end{array}$ & $\stackrel{3}{\%}$ & $\stackrel{\%}{\%}$ & $\begin{array}{l}\text { FRONTER } \\
\text { NUMERO } \\
\text { CASOS }\end{array}$ & $\begin{array}{c}\text { A NORTE } \\
\text { TOTAL } \\
\%\end{array}$ \\
\hline Hasta 350 dlls. & 7 & 3.1 & 1 & 24 & 10.8 & 3.6 & 28 & 12.6 & 4.2 & 59 & 8.8 \\
\hline De 351 a 750 & 43 & 19.4 & 6.5 & 61 & 27.5 & 9.2 & 59 & 26.6 & 8.8 & 163 & 24.5 \\
\hline De 751 a 950 & 45 & 20.3 & 6.8 & 21 & 9.5 & 3.1 & 26 & 11.7 & 3.9 & 92 & 13.8 \\
\hline De 951 a 1150 & 27 & 12.2 & 4 & 15 & 6.7 & 2.3 & 11 & 5 & 1.6 & 53 & 7.9 \\
\hline De 1151 a 1350 & 23 & 10.4 & 3.4 & 15 & 6.7 & 2.3 & 12 & 5.4 & 1.8 & 50 & 7.5 \\
\hline De 1351 a 1550 & 14 & 6.3 & 2.1 & 2 & 0.9 & 0.3 & 4 & 1.8 & 0.6 & 20 & 3 \\
\hline De 1551 a 1750 & 14 & 6.3 & 2.1 & 6 & 2.7 & 0.9 & 3 & 1.3 & 0.5 & 23 & 3.5 \\
\hline De 1751 a 1950 & 7 & 3.1 & 1.1 & 3 & 1.4 & 0.4 & 0 & 0 & 0 & 10 & 1.5 \\
\hline De 1951 a 2150 & 7 & 3.2 & 1.1 & 1 & 0.5 & 0.1 & 0 & 0 & 0 & 8 & 1.2 \\
\hline De 2151 a 2350 & 0 & 0 & 0 & 0 & 0 & 0 & 0 & 0 & 0 & 0 & 0 \\
\hline De 2351 a 2550 & 3 & 1.3 & 0.5 & 0 & $\theta$ & 0 & 2 & 0.9 & 0.3 & 5 & 0.8 \\
\hline De 2551 y más & 3 & 1.3 & 0.5 & 4 & 1.8 & 0.6 & 2 & 0.9 & 0.3 & 9 & 1.4 \\
\hline No contestó & 11 & 5 & 1.6 & 22 & 9.9 & 3.3 & 11 & 5 & 1.7 & 44 & 6.6 \\
\hline TOTAL & 222 & 100 & 33.4 & 222 & 100 & 33.3 & 222 & 100 & 33.3 & 666 & 100 \\
\hline
\end{tabular}

FUENTE: Encuesta del proyecto "Encuesta sobre transmigración en la frontera norte", Instituto de Investigaciones Sociales UABC, aplicada en Tijuana, Mexicali, San Luis R.C., Nogales, Ciudad Juárez, Piedras Negras, Nuevo Laredo, Reynosa y Matamoros, en febreromarzo de 1987; con una muestra de 666 casos. 
de negocios de la frontera sur de Estados Unidos. ${ }^{15}$ Otro antecedente que cabe mencionar es el del Programa de Braceros, que tuvo como objetivo ampliar la oferta de mano de obra - escasa por la Segunda Guerra Mundial- en la Unión Americana; este programa fortaleció notablemente al grupo de commuters, debido a que ". . . el número de éstos se incrementó rápidamente durante los años del programa de braceros. Los empleadores norteamericanos ayudaron a los mejores elementos de la fuerza de trabajo (braceros) a obtener visas de inmigrantes con estatus de residentes permanentes. Estos trabajadores se convirtieron en commuters porque mantuvieron su domicilio en México y trabajaban en Estados Unidos, constituyendo una fuente de trabajadores agrícolas cerca de la frontera. Esta fuente disponible de fuerza laboral jugó un rol importante en la terminación del programa de braceros". ${ }^{16}$ Un dato que ilustra la importancia de los trabajadores agrícolas en aquellos años, es el proporcionado por los resultados de la encuesta aplicada por el SIN, que muestra que en 1967 el $40 \%$ de los commuters declararon estar trabajando en actividades agrícolas. ${ }^{17}$

En la actualidad, la composición de la fuerza de trabajo según la rama de actividad ha cambiado - de acuerdo con la transformación de la economía del sur de los Estados Unidos-en favor de los sectores industrial, comercial y de servicios; esto se manifiesta en cierta forma, en los resultados obtenidos en la "Encuesta sobre Transmigración en la Frontera Norte", ya que el $\mathbf{2 2 . 8 \%}$ del total de commuters labora en el sector industrial (transformación, construcción y maquila); el $21.9 \%$ en el sector servicios (educación, domésticos, técnicos y profesionales); el $21.4 \%$ en el comercio; y el $17.3 \%$ en el sector agrícola (ver cuadro 2 ).

Comparando estos datos con los de la estructura del empleo del sur de los Estados Unidos, encontramos que el número de commuters agrícolas es verdaderamente alto, ya que en 1970 la fuerza de trabajo de los estados fronterizos del sur de la Unión Americana dedicada a esta actividad era el $3.7 \%$, y para 1980 el $3.0 \% .^{18}$ Sin embargo, en nuestro caso, casi una quinta parte son trabajadores agricolas. Este es un claro indicador de que la demanda de trabajo de este sector continua cubierta - además de los trabajadores indocumentados- por este grupo, que no se ha despojado de su función tradicionalmente agrícola en el mercado de trabajo estadounidense.

La incorporación de esta fuerza de trabajo en la estructura ocupacional norteamericana, está dirigida a los escalones más amplios y bajos de la pirámide ocupacional, aunque esto no incluye a todos los commuters. Es necesario precisar aquí que no se está hablando de un grupo

15 Report of the Select. . . op. cit. p. 108.

16 Ibid. p. 104.

17 Immigration and Naturalization. . op. cit. p. 19.

18 U.S. Census of Population. 1970, 1980. 
CUADRO 2. Número y porcentaje de commuters según región (1, 2 y 3) y frontera norte total por actividad que desempeña.

\begin{tabular}{|c|c|c|c|c|c|c|c|c|c|c|c|}
\hline ACTIVIDAD REGION & $\begin{array}{l}\text { NUMERO } \\
\text { CASOS }\end{array}$ & $\begin{array}{c}1 \\
\% \\
\text { REGION }\end{array}$ & $\begin{array}{c}\% \\
\text { Tớ }\end{array}$ & $\begin{array}{c}\text { NUMERO } \\
\text { CASUS }\end{array}$ & $\begin{array}{c}2 \\
\% \\
\text { REGION }\end{array}$ & $\begin{array}{c}\% \\
\text { TOTAL }\end{array}$ & $\begin{array}{c}\text { NUMERO } \\
\text { CASOS }\end{array}$ & $\begin{array}{c}3 \\
\% \\
\text { REGION }\end{array}$ & $\begin{array}{c}\% \\
\text { TOTAL }\end{array}$ & $\begin{array}{l}\text { FRONTER } \\
\text { NUMERO } \\
\text { CASOS }\end{array}$ & $\begin{array}{c}\text { A NORTE } \\
\% \\
\text { TOTAL }\end{array}$ \\
\hline
\end{tabular}

Agricultura, silvicultura, ganadería y caza

Pesca

Explotación de minas y canteras

Industria de transformación

Industria maquiladora

Construcción

Generación y distribución

de energía eléctric

Comereio

Transporte

Gobierno

Establecimientos financieros.

seguros, bienes inmuebles

Turismo (hoteles, restaurantes)

Servieios de educación

Servicios domésticos

Servicios técnicos y profesionales

Otros servicios

Actividades insuficientemente

especificadas

No trabaja

\begin{tabular}{|c|c|c|c|c|c|c|c|c|c|c|}
\hline 85 & 38.3 & 12.8 & 6 & 2.7 & 0.9 & 24 & 10.8 & 3.6 & 115 & 17.3 \\
\hline 2 & 0.9 & 0.3 & 1 & 0.4 & 0.2 & 5 & 2.2 & 0.7 & 8 & 1.2 \\
\hline 0 & 0 & 0 & 0 & 0 & 0 & 0 & 0 & 0 & 0 & 0 \\
\hline 9 & 4 & 1,3 & 9 & 4 & 1.3 & 3 & 1.3 & 0.5 & 21 & 3.1 \\
\hline 14 & 6.3 & 2.1 & 28 & 12.6 & 4.2 & 8 & 3.6 & 1.2 & 50 & 7.5 \\
\hline 15 & 6.7 & 2.3 & 24 & 10.8 & 3.6 & 39 & 17.6 & 5.8 & 78 & 11.7 \\
\hline 2 & 0.9 & 0.3 & 1 & 0.5 & 0.2 & 0 & 0 & 0 & 3 & 0.5 \\
\hline 17 & 7.6 & 2.6 & 31 & 14 & 4.6 & 53 & 23.9 & 8 & 101 & 15.2 \\
\hline 3 & 1.3 & 0.4 & 12 & 5.4 & 1.8 & 10 & 4.5 & 1.5 & 25 & 3.7 \\
\hline 5 & 2.3 & 0.8 & 3 & 1.3 & 0.4 & 8 & 3.6 & 1.2 & 16 & 2.4 \\
\hline 7 & 3.2 & 1 & 1 & 0.5 & 0.2 & 2 & 0.9 & 0.3 & 10 & 1.5 \\
\hline 7 & 3.2 & 1.1 & 23 & 10.4 & 3.4 & 11 & 5 & 1.7 & 41 & 6.2 \\
\hline 5 & 2.3 & 0.7 & 4 & 1.8 & 0.6 & 4 & 1.8 & 0.6 & 13 & 1.9 \\
\hline 5 & 2.3 & 0.7 & 13 & 5.9 & 2 & 14 & 6.3 & 2.1 & 32 & 4.8 \\
\hline 18 & 8.1 & 2.7 & 28 & 12.6 & 4.2 & 16 & 7.2 & 2.4 & 62 & 93 \\
\hline 10 & 4.5 & 1.5 & 18 & 8.1 & 2.7 & 11 & 5 & 1.7 & 39 & 5.9 \\
\hline 10 & 4.5 & 1.5 & 4 & 1.8 & 0.6 & 9 & 4.1 & 1.4 & 23 & 3.5 \\
\hline 8 & 3.6 & 1.2 & 16 & 7.2 & 2.4 & 5 & 2.2 & 0.7 & 29 & 4.3 \\
\hline 222 & 100 & 33.3 & 222 & 100 & 33.3 & 222 & 100 & 33.4 & 666 & 100 \\
\hline
\end{tabular}

FUENTE: Encuesta del proyecto "Encuesta sobre transmigración en la frontera norte", aplicada en Tijuana, Mexicali, San Luis R.C., Nogales, Ciudad Juárez, Piedras Negras, Nuevo Laredo, Reynosa y Matamoros, en febrero-marzo de 1987; con una muestra de 666 casos. Instituto de Investigaciones Sociales de la UABC. 
homogéneo y uniforme, sino de un amplio sector que conjunta historias disímiles y posiciones sociales diversas en el contexto ocupacional fronterizo; sin embargo, la mayoría de esta fuerza de trabajo tiene como ocupación principal la de obrero o jornalero $(44.3 \%)$, empleados domésticos (16.9\%), vendedores dependientes y oficinistas (12.9\%). Estos grupos en conjunto representan casi tres cuartas partes del total; en cambio, el personal profesional $(1.9 \%)$, técnicos especializados $(4.5 \%)$, maestros y afines (1.5\%), funcionarios públicos $(0.1 \%)$ y gerentes del sector privado (1.9\%) -las cuales son ocupaciones de mayor jerarquia en la estructura ocupacional-, suman el $9.9 \%$ (ver cuadro 3 ). Otro dato que ilustra con claridad este panorama es el hecho de que el $86.6 \%$ declararon ser empleados, jornaleros y obreros; $5.7 \%$ mayordomos, $4.1 \%$ trabajadores por su cuenta y $2.9 \%$ patrones o empleadores (ver cuadro 4 ).

Ya se ha mencionado, en términos de ingreso, que el trabajador commuter tiene un estatus privilegiado en relación con la población fronteriza de México. Un elemento que sustenta esta afirmación deriva del hecho de que perciben su salario en dólares, lo cual produce un cambio proporcional al transformarlo en pesos. Así, los ingresos de éstos prácticamente se disparan en comparación con los salarios nominales -no se diga salarios reales - en la frontera mexicana. Revisemos algunos datos: la mediana del ingreso mensual declarado por los commuters encuestados fue de 850 dólares; si se considera que la paridad cambiaria peso-dólar durante los primeros días de enero de 1987 era aproximadamente de 900 pesos por dólar, este ingreso se traduce en $\$ 765,000$ pesos que equivale a 8.3 veces el salario mínimo mensual de la zona III en México $(\$ 3,050$ pesos diarios). De esta manera, no obstante que en el contexto salarial norteamericano este ingreso es bajo, en México resulta muy elevado (véase cuadro 1). Por otro lado, el gasto de sus ingresos, según se efectúe en México o en Estados Unidos, denota una clara inclinación a realizar la mayor parte de sus compras en donde les resulta más redituable, en este caso en México. Por ejemplo, sólo el 20.4\% declararon gastar más del 50\% de sus ingresos en Estados Unidos; el 17.9\% manifestó gastar en proporciones similares en México y en Estados Unidos; y el 61.7\% afirmó gastar más de la mitad de sus ingresos en la frontera mexicana.

\section{CONCLUSIONES}

Los trabajadores commuters han actuado en la frontera MéxicoEstados Unidos desde que aparece el control de la línea internacional. Su integración al mercado laboral norteamericano, históricamente ha respondido a las necesidades de fuerza de trabajo ahí generadas, así como a la falta de opciones de trabajo en México que sean competitivas con las que se presentan del otro lado de la frontera. Así, algunos sectores productivos del sur de Estados Unidos han cubierto su demanda de mano 
CUADRO 3. Número y porcentaje de commuters según región (1, 2 y 3) y frontera norte total por ocupación principal.

\begin{tabular}{|c|c|c|c|c|c|c|c|c|c|c|c|}
\hline $\begin{array}{l}\text { OCUPACION } \\
\text { PRINCIPAL }\end{array}$ & $\begin{array}{c}\text { NUMERO } \\
\text { CASOS }\end{array}$ & $\begin{array}{c}1 \\
\% \\
\text { HEGION }\end{array}$ & $\begin{array}{c}\% \\
\text { Turial. }\end{array}$ & $\begin{array}{l}\text { NUMERO } \\
\text { CASOS }\end{array}$ & $\begin{array}{c}2 \\
\% \\
\text { REGION }\end{array}$ & $\stackrel{x}{\text { TOTAL }}$ & $\begin{array}{c}\text { NUMERO } \\
\text { CASOS }\end{array}$ & $\begin{array}{c}3 \\
\% \\
\text { HEGION }\end{array}$ & $\begin{array}{c}\% \\
\text { TOYTAL }\end{array}$ & $\begin{array}{l}\text { FRONTER } \\
\text { NUMERO } \\
\text { CASOS }\end{array}$ & $\begin{array}{l}\text { A NORTE } \\
\% \\
\text { TUTAL. }\end{array}$ \\
\hline Profesionales & 6 & 2.7 & 0.9 & 5 & 2.3 & 0.7 & 2 & 0.9 & 0.3 & 13 & 1.9 \\
\hline Téenieos y personal especializado & 7 & 3.2 & 1.1 & 12 & 5.4 & 1.8 & 11 & 5 & 1.6 & 30 & 4.5 \\
\hline Meestros y afines & 3 & 1.3 & 0.4 & 2 & 0.9 & 0.3 & 5 & 2.2 & 0.8 & 10 & 1.5 \\
\hline Trabajadores del arte & 0 & 0 & 0 & 1 & 0.5 & 0.1 & 0 & 0 & 0 & 1. & 0.1 \\
\hline Funcionarios públicos & 1 & 0.4 & 0.1 & 2 & 0.9 & 0.3 & 4 & 1.8 & 0.6 & 7 & 1 \\
\hline Gerentes scetor privado & 3 & 1.4 & 0.5 & 1 & 0.5 & 0.1 & 9 & 4 & 1.3 & 13 & 1.9 \\
\hline Administrador agropecuario & 0 & 0 & 0 & 0 & 0 & 0 & 0 & 0 & 0 & 0 & 0 \\
\hline Mayorales agropecuarios & 1 & 0.4 & 0.2 & 0 & 0 & 0 & 0 & 0 & 0 & 1 & 0.2 \\
\hline Agricultores & 48 & 21.6 & 7.2 & 3 & 1.3 & 0.5 & 13 & 5.9 & 1.9 & 64 & 9.6 \\
\hline Op. de maquinaria agropecuaria & 4 & 1.8 & 0.6 & 0 & 0 & 0 & 1 & 0.5 & 0.1 & 5 & 0.7 \\
\hline Supervisores de obreros & 8 & 3.6 & 1.2 & 4 & 1.8 & 0.6 & 2 & 0.9 & 0.3 & 14 & 2.1 \\
\hline Artesanos y obreros & 86 & 38.7 & 12.9 & 78 & 35.1 & 11.7 & 65 & 29.3 & 9.8 & 229 & 34.4 \\
\hline Ayudantes de obreros & 0 & 0 & 0 & 0 & 0 & 0 & 2 & 0.9 & 0.3 & 2 & 0.3 \\
\hline Oficinistas & 6 & 2.7 & 0.9 & 6 & 2.7 & 0.9 & 8 & 3.6 & 1.2 & 20 & 3 \\
\hline Vendedores dependientes & 10 & 4.5 & 1.5 & 22 & 9.9 & 3.3 & 34 & 15.3 & 5.1 & 66 & 9.9 \\
\hline Vendedores ambulantes & 2 & 0.9 & 0.3 & 0 & 0 & 0 & 3 & 1.3 & 0.5 & 5 & 0.8 \\
\hline \multicolumn{12}{|l|}{ Empleados de servicios } \\
\hline domésticos & 14 & 6.3 & 2.1 & 36 & 16.2 & 5.4 & 35 & 15.8 & 5.3 & 85 & 12.8 \\
\hline Trabajadores domésticos & 4 & 1.8 & 0.6 & 12 & 5.4 & 1.8 & 11 & 5 & 1.7 & 27 & 4.1 \\
\hline Operador de transporte & 3 & 1.4 & 0.5 & 10 & 4.5 & 1.5 & 5 & 2.2 & 0.7 & 18 & 2.7 \\
\hline Proteceión y vigilancia & 0 & 0 & 0 & 1 & 0.5 & 0.2 & 0 & 0 & 0 & 1 & 0.2 \\
\hline No especificado & 9 & 4.1 & 1.4 & 8 & 3.6 & 1.2 & 2 & 0.9 & 0.3 & 19 & 2.9 \\
\hline Nunca ha trabajado & 7 & 3.2 & 1 & 19 & 8.5 & 2.9 & 10 & 4.5 & 1.5 & 36 & 5.4 \\
\hline TOTAI. & 222 & 100 & 33.4 & 222 & 100 & 33.3 & 222 & 100 & 33.3 & 666 & 100 \\
\hline
\end{tabular}

FUENTE: Encuesta del proyecto "Encuest a sobre transmigración en la frontera norte", aplicada en Tijuana. Mexicali, San Luis R.C.. Nogales, Ciudad Juárez, Piedras Negras, Nuevo Laredo, Reynosa y Matamoros, en febrero-marzo de 1987; con una muestra de 666 casos. Instituto de Investigaciones Sociales de la UABC. 
CUADRO 4. Número y porcentaje de commuters según región $(1,2$ y 3$)$ y frontera norte total por posición ocupacional.

\begin{tabular}{|c|c|c|c|c|c|c|c|c|c|c|c|}
\hline $\begin{array}{ll} & \text { REGION } \\
\text { POSICION } & \\
\text { OCUPACIONAL } & \end{array}$ & $\begin{array}{c}\text { NUMERO } \\
\text { CASOS }\end{array}$ & $\begin{array}{c}1 \\
\text { REGION }\end{array}$ & TOT:AL & $\begin{array}{c}\text { NUMERO } \\
\text { CASOS }\end{array}$ & $\begin{array}{c}2 \\
\% \\
\text { REGION }\end{array}$ & TOTAL & $\begin{array}{l}\text { NUMERO } \\
\text { CASOS }\end{array}$ & $\begin{array}{c}3 \\
\% \\
\text { REGION }\end{array}$ & TOV'TลL & $\begin{array}{l}\text { FRONTER } \\
\text { NUMERO } \\
\text { CASOS }\end{array}$ & $\begin{array}{c}\text { A NORTE } \\
\% \\
\text { TOTTAL }\end{array}$ \\
\hline Empleado & 143 & 64.4 & 21.5 & 155 & 69.8 & 23.3 & 139 & 62.6 & 20.9 & 437 & 65.6 \\
\hline Obrero o jornalero & 55 & 24.8 & 8.3 & 30 & 13.5 & 4.5 & 55 & 24.8 & 8.2 & 140 & 21 \\
\hline Mayordomo & 8 & 3.6 & 1.2 & 17 & 7.7 & 2.6 & 13 & 5.9 & 1.9 & 38 & 5.7 \\
\hline Trabaja por su cuenta & 9 & 4 & 1.3 & 10 & 4.5 & 1.5 & 8 & 3.6 & 1.2 & 27 & 4.1 \\
\hline Patrón o empleador & 5 & 2.3 & 0.7 & 9 & 4.1 & 1.3 & 5 & 2.2 & 0.8 & 19 & 2.9 \\
\hline No contestó & 2 & 0.9 & 0.3 & 1 & 0.5 & 0.2 & 2 & 0.9 & 0.3 & 5 & 0.7 \\
\hline TOTAL & 222 & 100 & 33.3 & 222 & 100 & 33.4 & 222 & 100 & 33.3 & 666 & 100 \\
\hline
\end{tabular}

FUENTE: Encuesta del proyecto "Encuesta sobre transmigración en la frontera norte", aplicada en Tijuana, Mexicali, San Luis R.C., Nogales, Ciudad Juárez, Piedras Negras, Nuevo Laredo, Reynosa y Matamoros, en febrero-marzo de 1987; con una muestra de 666 casos. Instituto de Investigaciones Sociales de la UABC. 
de obra con una gran parte de este grupo de trabajadores; es decir, con aquéllos que no han podido colocarse en puestos con niveles de ingresos medios $o$ altos, y que dentro de la escala salarial estadounidense reciben muy bajos ingresos - fundamentalmente los del estado de Texas.

Por otro lado, es válido suponer que una de las razones por las cuales los commuters mantienen su residencia en México esté en relación con el papel que juegan en el mercado laboral norteamericano, que les confiere empleos mal remunerados, no aceptados por la población nativa (como es el caso del trabajo agrícola), que además requieren de un intenso desgaste físico, y proporcionan poco estatus (como sucede con las trabajadoras domésticas). No obstante su ubicación en la estructura ocupacional estadounidense, el commuter disfruta actualmente de una posición económica más favorable que la población fronteriza asalariada de México, derivada del deslizamiento de la moneda mexicana en relación con el dólar; incluso en las localidades fronterizas mexicanas los sectores medios (maestros, médicos, profesionistas diversos, etc.) que no se vinculaban al flujo de emigrantes hacia Estados Unidos, ante un deterioro salarial inusitado, están optando por buscar fuentes de trabajo en el país vecinoo en otro sector de la economía fronteriza como por ejemplo el comercio.

De esta manera, el commuter - a pesar de su posición en la Unión Americana-constituye un sector que permanece en México con un alto nivel de ingresos, $y$ que dinamiza las economías locales al inyectar divisas; sin embargo, también propicia la inflación al provocar una demanda en dólares en las localidades fronterizas mexicanas, que activa el flujo de circulante en estas regiones como resultado de los diferenciales en la relación cambiaria. La situación tan peculiar de este grupo de población fronteriza tan sólo denota uno de los grandes contrastes que se manifiestan en la frontera México-Estados Unidos. 


\section{BIBLIOGRAFIA}

ACUÑ̃ González, Beatriz G., y otros. El trabajador mexicano en Estados Unidos; transmigración y desarrollo en la frontera Tijuana-San Diego. Mexicali, B.C. IIS.UABC. 1984.

ARAMBURO V., Guillermo. Transmigración en la frontera Tijuana-San Diego. El caso de los trabajadores agrícolas indocumentados. Tesis. El Colegio de la Frontera Norte. Tijuana, B.C. 1986.

BUSTAMANTE, Jorge A. Espaldas mojadas: materia prima para la expansión del capital norteamericano. México, El Colegio de México. 1976 (Cuadernos del CES. 9).

CLEMENT, Norris. "Perspectiva sobre el desarrollo económico de la región fronteriza del suroeste de los Estados Unidos". En: Administración del desarrollo de la frontera norte. Ed. Mario Ojeda, México, El Colegio de México. 1982.

ERICKSON, Anna-Stina. "The impact of commuters on the MexicanAmerican border area". Monthly Review. August, 1970.

GORDON, Charles. "The Amiable Fiction-Alien Commuters under or Inmigration Laws". Hearings before the Special Subcommitte on Labor House Representative. Ninety First Congrenss. Washington, D.C., July, 1969.

MARGULIS M., y Rodolfo Tuirán. Desarrollo y población en la frontera norte: el caso de Reynosa. México, El Colegio de México. 1986.

NORTH S., David and Marion F. Houston. The Characteristics and Role of Illegal Aliens in the U.S. Labor Market: An exploratory study. Washington, Linton and Company, 1976.

RUNGELIA, B.C. Impact of the Mexican Alien commuter on the apparel industry of El Paso, Texas (case study). Thesis, Kentucky. 1969. Report of the Select Commission on Western Hemisphere Inmigration. Washington, January, 1968.

"The Commuter on the United States-Mexico Border". United States Commission on Civil Rights Staff Report. Hearings before the Special Subcommitte on Labor House Representative. Ninety First Congress. Washington, D.C., July, 1969. 